\title{
Aplicación móvil "Applndustria 4.0": una herramienta para la evaluación de las organizaciones en industria 4.0
}

\author{
Marco A. Díaz-Martínez ${ }^{1 *}$, Santos Ruíz-Hernández ${ }^{1}$, Reina V. Román-Salinas ${ }^{1}$, y Gabriela Estrada-Cadena ${ }^{2}$ \\ (1) Tecnológico Nacional de México, Campus Pánuco (ITSP), México. (correo-e: marco.dm@panuco.tecnm.mx; \\ santos.rh@panuco.tecnm.mx; reina.rs@panuco.tecnm.mx) \\ (2) Tecnológico de Monterrey, México. (correo-e: gabriela.estrada@tec.mx) \\ *Autor a quien debe ser dirigida la correspondencia
}

Recibido Nov. 19, 2020; Aceptado Ene. 15, 2021; Versión final Mar. 1, 2021, Publicado Ago. 2021

\begin{abstract}
Resumen
El objetivo de este artículo es presentar una aplicación móvil, Applndustria 4.0, que permite evaluar el nivel de implementación de la Industria 4.0 en las organizaciones. La aplicación ésta conformada por módulos gestionados de forma centralizada a través de variables y está desarrollada íntegramente bajo la plataforma Android. La metodología consta del desarrollo y funcionamiento de software, respuestas ante cambios del software, colaboración con cliente sobre el uso del software, interacción con individuos e interacciones sobre los procesos y herramientas. La Applndustria 4.0 se aplicó a 50 personas con responsabilidad gerencial con conocimiento en procesos organizacionales. Las variables no presentan multicolinealidad y se tiene una fiabilidad del Alfa de Cronbach de 0.85 . Existe una fuerte relación de contribución de las variables proceso $(24.87 \%)$, personas $(24.11 \%)$ y tecnología $(42.33 \%)$. Se concluye que la Applndustria 4.0 es factible de utilizar para las organizaciones y se acreditó las pruebas estadísticas de confiabilidad para su uso y aplicación.
\end{abstract}

Palabras clave: industria 4.0; dispositivo móvil; organización; convergencia digital; tecnología

\section{The mobile application "Applndustria 4.0": a tool for evaluating organizations in Industry 4.0}

\begin{abstract}
The objective of this article is to present an Industry 4.0 mobile application, Applndustria 4.0, which allows evaluating the level of Industry 4.0 implementation among organizations. The application is composed of modules managed centrally through variables. The mobile application is developed entirely in Android. The methodology consists of: software development and operation, responses to software changes, client assistance for software use, interaction with individuals, and interactions of processes and tools. Fifty people with managerial responsibility and with knowledge of organizational processes test the Applndustria 4.0 app. The results show that the variables examined do not have multi-colinearity. The reliability of Cronbach's alpha is 0.85 . There is also a strong contribution by the process variables (24.87\%), people (24.11\%), and technology $(42.33 \%)$. It is concluded that the Applndustria 4.0 is reliable for its implementation as it is accredited by reliability statistical tests for use and application.
\end{abstract}

Keywords: industry 4.0; mobile device; organization; digital convergence; technology 


\section{INTRODUCCIÓN}

La industria 4.0 se inició en Alemania resultado de la transformación digital de la industria conocida como "Fábrica inteligente". El avance del internet tiene influencia diferentes sectores en la economía y en la sociedad; las tecnologías de información ( $\mathrm{TI}$ ) con uso de sensores y robótica, han transformado el internet de las cosas (IoT) en organizaciones empresariales (Bueno et al., 2020). Con tecnología las empresas reciben gran cantidad de información desde diferentes equipos automatizados, sistemas de control en tiempo real y análisis de sistemas de producción; la organización genera información de sus actividades cotidianas causando un incremento del proceso de digitalización y distribución de información de forma permanente, esto requiere un esfuerzo adicional del personal involucrado (Hsion et al., 2020). El desarrollo tecnológico tiene impacto en sistemas de manufactura, en mecanizado de procesos, la automatización y robótica; actualmente, con la "Industria 4.0" es considerada como la Cuarta revolución industrial. Este concepto tecnológico es considerado como sistema que redefine modelos de negocio y genere una perspectiva global que integre el entorno organizacional; la industria de la manufactura presenta cambios y transformaciones digitales que están revolucionado el sector que permiten a las empresas explotar datos y mejorar sus sistemas.

La cuarta revolución industrial es la mayor transformación que se ha conocido por la humanidad, ya que tiene un impacto en todas las actividades: la forma en que hacemos las cosas, como usamos los recursos, la interacción entre personas, como trabajamos, como aprendemos, como hacemos negocios; en este aspecto, se tiene en consideración un instrumento que puede medir la percepción de la Industria 4.0 de forma manual (Ruiz et al., 2019). En este contexto se presenta el siguiente desafío, pensar en una aplicación móvil, que más allá de su arquitectura tecnológica, permita construir un sentido claro y verdadero sobre cómo se encuentra la organización con respecto a la industria 4.0 mediante un sistema de evaluación organizacional para mejorar la toma mejores decisiones y adaptarse a esta nueva revolución industrial.

Una de las problemáticas que presentan las organizaciones, es la ausencia de un instrumento de evaluación organizacional que permita conocer las áreas de oportunidad y mejorar el nivel de implementación de la industria 4.0. En la actualidad, la información sobre la Industria 4.0 es recuperada por correo electrónico o en entrevistas cara a cara. La entrada de una aplicación que pueda medir la Industria 4.0, amplia el alcance de la información y potencializa sus resultados. El objetivo de este trabajo es presentar una aplicación móvil que permite medir el nivel de implementación de la industria 4.0 en las organizaciones, el instrumento es evaluado mediante ecuaciones estructurales PLS con XLSTAT para identificar la estructura interna de los datos; así mismo, se estableció las relaciones entre variables con la responsabilidad social a manera de ejemplo para la representación completa del estudio.

Existen más de trescientas mil aplicaciones para dispositivos móviles; en este sentido, Android lidera las descargas de aplicaciones inteligentes. En este sentido se pueden encontrar aplicaciones formales e informales de carácter público y privado. En la actualidad, lo sistemas son en ambiente web y permite utilizar las herramientas, almacenaje y acceso a la nube donde este último es considerado como un elemento de beneficio para industria 4.0 (Garita, 2013). El desarrollo experimentado las tecnologías de información han generado nuevas plataformas digitales e infraestructura, logrando con ello la globalización y la eficiencia de los procesos en diferentes áreas de la sociedad, relacionados con el intercambio de información y conocimiento (Chavira y Arredondo, 2017).

\section{OTROS ANTECEDENTES}

Las variables de estudio de esta investigación que permiten medir el nivel de implementación de la industria 4.0 en las organizaciones son: Procesos, Productos, Personas, Tecnología, Negocios y una variable de salida Responsabilidad social. Para lograr esto se construye las siguientes relaciones.

\section{Procesos-Responsabilidad social}

La automatización ha ido evolucionando mediante el uso de computadoras en los procesos de manufactura y la industria 4.0 se están introduciendo en nuevos retos y desafíos que necesitan ser resueltos. Algunos de estos retos están relacionados con la integración de actores heterogéneos que puedan llevar las tareas de los procesos de manufactura. Además, es crucial como la industria 4.0 enfocada al análisis de los procesos pueda permitir que los trabajadores tengan una relación optima con los procesos de producción (Sánchez et al., 2020). Por otro lado, la innovación de procesos que se implementan en las empresas a través de la industria 4.0 han impactado a favor de la innovación de la cadena de suministro como primer orden y un efecto positivo en segundo orden sobre el desempeño económico a largo plazo donde las empresas pueden obtener mayor rendimiento colaborando con los proveedores en programas ambientales o invirtiendo en tecnología 4.0 pero no con ambas (De Giovanni y Cariola, 2020). Dada esta perspectiva se propone la siguiente hipótesis. $\mathrm{H} 1$ : Los procesos tienen influencia positiva en la responsabilidad social 


\section{Personas-Responsabilidad social}

La transición a la industria 4.0 y el aumento de la ciberfísica donde los sistemas están introduciendo aspectos técnicos y organizativos que están relacionados con las personas en una organización. La importancia de contar con la seguridad de los trabajadores al trabajar con tecnologías de automatización tangibles como robots y vehículos autónomos ha ido mejorando cada día con la entrada de la industria 4.0 (Kadir y Broberg, 2021). Por otro lado, Sony y Naik (2020), argumentan que el concepto de la industria 4.0 existe un sistema socio-técnico. Por lo tanto, para tener una implementación sostenible de las tecnologías habilitadoras de la industria 4.0 y adaptarse para lograr el bienestar de los trabajadores, es necesario aplicar sistemas con perspectiva de estudios sociotécnicos.

Para Gaudencio et al. (2017), indica que los trabajadores que laboran en empresas socialmente responsables tienen un comportamiento positivo con la organización, perciben que es confiable y se identifican con ella. Se complementa con la satisfacción laboral, la motivación e intenciones de rotación del personal. Dada esta perspectiva se propone la siguiente hipótesis. H2: Las Personas tienen influencia positiva en la responsabilidad social

\section{Tecnología-Responsabilidad social}

La industria 4.0 está revolucionando la fabricación, aumentando la flexibilidad, la personalización en masa, calidad y la productividad. En la fabricación actual, el mantenimiento es uno de los temas más críticos y las empresas se están acercando a su transformación digital desde una perspectiva tecnológica y de gestión (Silvestri et al. 2020). Estas nuevas tecnologías que vienen incluidas en esta industria 4.0, ofrecen una oportunidad vital y valiosa para impulsar las redes sociales e innovaciones tecnológicas. Cuentan con una arquitectura tecnológica y sistemas ciberfísicos de última generación, que pueden aumentar drásticamente la productividad y el rendimiento de innovación de una empresa (Faraz y Petraite, 2020).

Con el tiempo la responsabilidad social ha ido ganando importancia y las empresas están tratando de encontrar un equilibrio adecuado entre la rentabilidad económica y responsabilidad social. A fin de que se descubra cuáles son las principales políticas, estrategias y prácticas que se han desarrollado en materia de responsabilidad social corporativa y como evaluarla (Fernández et al., 2015). Dada esta perspectiva se propone la siguiente hipótesis. H3: La tecnología tienen influencia positiva en la responsabilidad social

\section{Negocios-Responsabilidad social}

Cuando hablamos de la empresa inteligente es hablar de la tecnología avanzada en la industria tradicional. Un número cada vez mayor de infracciones de ciberseguridad afectan negativamente el rendimiento empresarial, al favorecerse de las vulnerabilidades de los sistemas de producción que están interconectadas mediante redes industriales. Este tipo de ataques pueden afectar de gran manera el modelo empresarial corporativo y es de suma importancia que al implementar la industria 4.0 se evalúen de antemano los principales activos críticos a proteger de posibles ciberataques y los impactos empresariales que podrían ocurrir es una fuerte ventaja competitiva (Corallo et al., 2020).

Para la sostenibilidad, las empresas deben adoptar una producción más limpia y realizar prácticas económicas para producir productos de mejor calidad al menor costo. La implantación de estas prácticas es altamente influenciada por los habilitadores de la industria 4.0 dentro del contexto ético y desarrollo empresarial sostenible (Shayganmehr et al., 2021). El concepto de responsabilidad social (RSE) acoge ideas afines al funcionamiento de las empresas en el marco del desarrollo del sistema económico-social. La RSE afianza la naturaleza y el quehacer de la empresa en un mundo cada vez más competitivo. Cabe mencionar que la RSE se conformó como concepto a partir de determinadas exigencias sociales y, concretamente, del mercado. Dada esta perspectiva se propone la siguiente hipótesis. H4: Los negocios tiene tecnología tienen influencia positiva en la responsabilidad social

\section{METODOLOGÍA}

La investigación realizada es cualitativa de tipo exploratorio para el desarrollo de la aplicación y fue de tipo exploratorio; se utilizó estadística inferencial para las pruebas de validez del instrumento de medición y fortalecer el análisis. Para el desarrollo de la aplicación se aplicaron métodos para el desarrollo de software existentes para ese motivo. Para presentar algunos datos se utilizó estadística descriptiva para la comprensión de los mismos. Existen diferentes metodologías para el desarrollo de aplicaciones móviles. La primera consta de cuatro fases 1) Desarrollo y funcionamiento de software; 2) Respuestas ante cambios del software; 3) Colaboración con cliente sobre el uso del software; 4) Interacción con individuos e interacciones sobre los procesos y herramientas; la segunda metodología existente llamada "6 M's" que deben su nombre a los seis atributos que evalúan el éxito del servicio propuesto: 1) Movement (Movimiento); 2) Moment (Momento); 3) Me (Yo); 4) Multi-user (Multiusuario); 5) Money (Dinero); 6) Machines (Máquinas) (Beck, et al., 2001). 
La metodología utilizada en esta investigación se adecua perfectamente y se encuentra enmarcada en cinco etapas descritas como: etapa 1) Análisis, se realizan entrevistas al cliente, para que manifieste las necesidades que se pretenden solucionar; etapa 2) Diseño, se cuenta con diferentes escenarios de conexión y sincronización con el servidor; etapa 3) Desarrollo, se programa en el lenguaje seleccionado de cada parte en la fase de diseño y se verifica el funcionamiento de la aplicación; 4) Pruebas de funcionamiento, verificación del funcionamiento de la aplicación en diferentes escenarios y condiciones, se realizan mediante la simulación; 5) Entrega, se finaliza la aplicación móvil y se entrega del ejecutable, el código fuente y manuales del sistema. Las etapas descritas son utilizadas en aplicaciones móviles (Mantilla et al., 2014).

Los participantes fueron seleccionados de forma aleatoria, de tal forma que reunieran los requisitos del objetivo de la investigación, es decir, personas con alto conocimiento sobre los procesos organizacionales y con responsabilidades en la toma decisiones en la empresa. El estudio se realizó con la participación de personas en diferentes estados de la república mexicana, como Tamaulipas, Estado de México, Veracruz, Querétaro y algunos municipios como Toluca, Tlalnepantla, Naucalpan. La muestra quedó conformada por 80 representantes de diferentes organizaciones que participaron con la Apps de la Industria 4.0.

\section{Desarrollo de Aplicación móvil}

Para desarrollar la aplicación se muestra el trabajo desarrollado en las etapas siguientes. Primera etapa: buscar y seleccionar un entorno adecuado para el desarrollo de la aplicación móvil; se evaluaron diferentes plataformas, se decidió que fuera Android Studio el software indicado para realizar la programación, ya que esta plataforma contiene una licencia de software libre. Segunda etapa: seleccionar el origen de los datos, se evaluaron diferentes servidores de datos, se decidió utilizar herramientas gratuitas ofrecidas por Google como la creación y diseño de formularios. Tercera etapa: Fase de análisis, diseño, formateo y programación. Esta etapa es la más importante que debe cumplir la aplicación para desempeñarse en forma adecuada, método fundamental para este tipo de aplicación.

El cumplimiento del objetivo planteado no dependerá de la aplicación móvil en sí misma, se hará realidad cuando se logren cumplir con varios aspectos, tales como: tener distribución masiva de la aplicación, que el uso de dispositivos móviles sean más eficientes y contar buena velocidad de internet. En la actualidad contar con nuevos canales de difusión que puedan utilizar tecnología móvil es una ventaja importante para llegar a cualquier organización que desee evaluarse en la industria 4.0. El uso de aplicaciones informáticas ejecutadas desde teléfonos inteligentes (App), es una realidad que forma parte del proceso cotidiano de la vida y que están presentes en la educación e investigación, específicamente en la gestión de información personal, laboral y académica; las tecnologías han sido adoptadas mucho más rápido por las nuevas generaciones, ya que, ellos prácticamente crecieron con ella; esta generación es conocida como la generación App; los jóvenes de ahora no solamente crecen con las tecnologías y aplicaciones, sino que entiende que están rodeados de un conjunto de aplicaciones, es decir que tienen presente que las tecnologías los seguirán para toda la vida.

El trabajo presentado se integra a partir las componentes de estudio indicadas a continuación: 1) Servidores de datos personal o terceros; 2) Características de la información, pública o privada; 3) Dispositivos a partir del cual se tiene acceso a la información; 4) Personal involucrado en diferentes tareas o actividades. El objetivo de este trabajo es presentar una aplicación móvil como instrumento de evaluación para medir el nivel de implementación de la industria 4.0 en las organizaciones. La construcción del instrumento incluyó la validez de contenido, de fiabilidad y confiabilidad a través de pruebas estadísticas. La aplicación cuenta con las siguientes variables de estudio: procesos, productos, personas, tecnología, negocios, y responsabilidad social.

Procesos. Determina el nivel en que se ubican los conocimientos de los procesos productivos o de servicios de la empresa, el nivel en que se realiza la supervisión de los procesos productivos, nivel de consideración adecuada de cada proceso productivo en la organización y también se considera que los procesos productivos o de servicios se pueden mejorar y que los procesos están armonizados para no afectar el medio ambiente.

Productos. Se considera que la mejora continua es un factor para incrementar el nivel de vida de un producto, la organización planea para mejorar los productos, el grado de implementación de la tecnología han mejorado los procesos de producción, la conectividad de dispositivos electrónicos en el área de producción mejora la fabricación de productos.

Personas. Se identifica si la conectividad del personal es adecuada en áreas de producción, se considera la capacitación adecuada del personal en uso de la tecnología en procesos de producción, se ubica el compromiso de las personas con las estrategias de mejora que implementa la empresa, identificar personal con alta especialización en manejo de tecnología empleada para producir y que la capacitación es una estrategia adecuada para adaptar el personal a las estrategias de negocio de la empresa. 
Tecnología. Si la organización está en condiciones para adquirir nueva tecnología para la mejora de la producción y competitividad, si están familiarizados con términos de tecnología, se identifica si la organización cuenta con presupuesto para la implementación de tecnología y si cuenta con medios electrónicos de difusión como diferenciador de competencia organizacional.

Negocios. Determina el nivel de implementación de la tecnología que facilite las compras de los clientes, hacer propuestas novedosas de base tecnológicas como ventaja competitiva, considerar que la industria 4.0 destaca como la base de la economía, que la tecnología debe ser parte estratégica de su modelo de negocio y que la organización debe crear nuevos productos, servicios y modelos de negocio orientados hacia un nuevo tipo de cliente.

Responsabilidad Social. En el estudio de esta variable podremos conocer si los procesos utilizados en la empresa ubican la importancia de no afectar el medio ambiente, el personal realiza actividades vinculadas con la cultura dentro y fuera de la empresa, si se destinan recursos orientados a sensibilizar la importancia del medio ambiente en los trabajadores, ubica si la empresa respeta las normatividades en materia de seguridad industrial, medio ambiente, residuos y si los desechos derivados de la producción están bajo medidas de control.

\section{Aplicación móvil como herramienta de difusión}

La evolución de la tecnología móvil es constante y avanza a gran velocidad, esto dificulta la comprensión del potencial que ofrecen este tipo de dispositivos y genera un reto entre los responsables de la toma de decisiones al interior de las empresas. La aplicación móvil que se describe no es una plataforma cerrada, su diseño modular permitirá añadir nuevas aplicaciones a través las actualizaciones necesarias para ampliar objetivos de investigación para estudios relacionados. La aplicación móvil se encuentra disponible desde el mes de septiembre del 2020 en la Play Store para su descarga y usabilidad. La Applndustria 4.0 inicia con una pantalla de bienvenida que identifica las instituciones desarrolladoras de la aplicación móvil. Una característica importante tiene que ver con la replicación de la aplicación, es decir, los datos seguirán alimentándose hasta que se cumpla el objetivo trazado de la investigación.

En la pantalla de bienvenida se encuentra un apartado para ingresar datos personales del informante y datos relacionados con el puesto que desempeña dentro de la organización. Esta acción ayuda a detectar la cantidad de usuarios que están utilizando la aplicación y recolectar la información de manera automática para su clasificación. No se van a presentar todas las pantallas de la aplicación, solamente alguna a manera de representativa de la aplicación (ver figura 1).

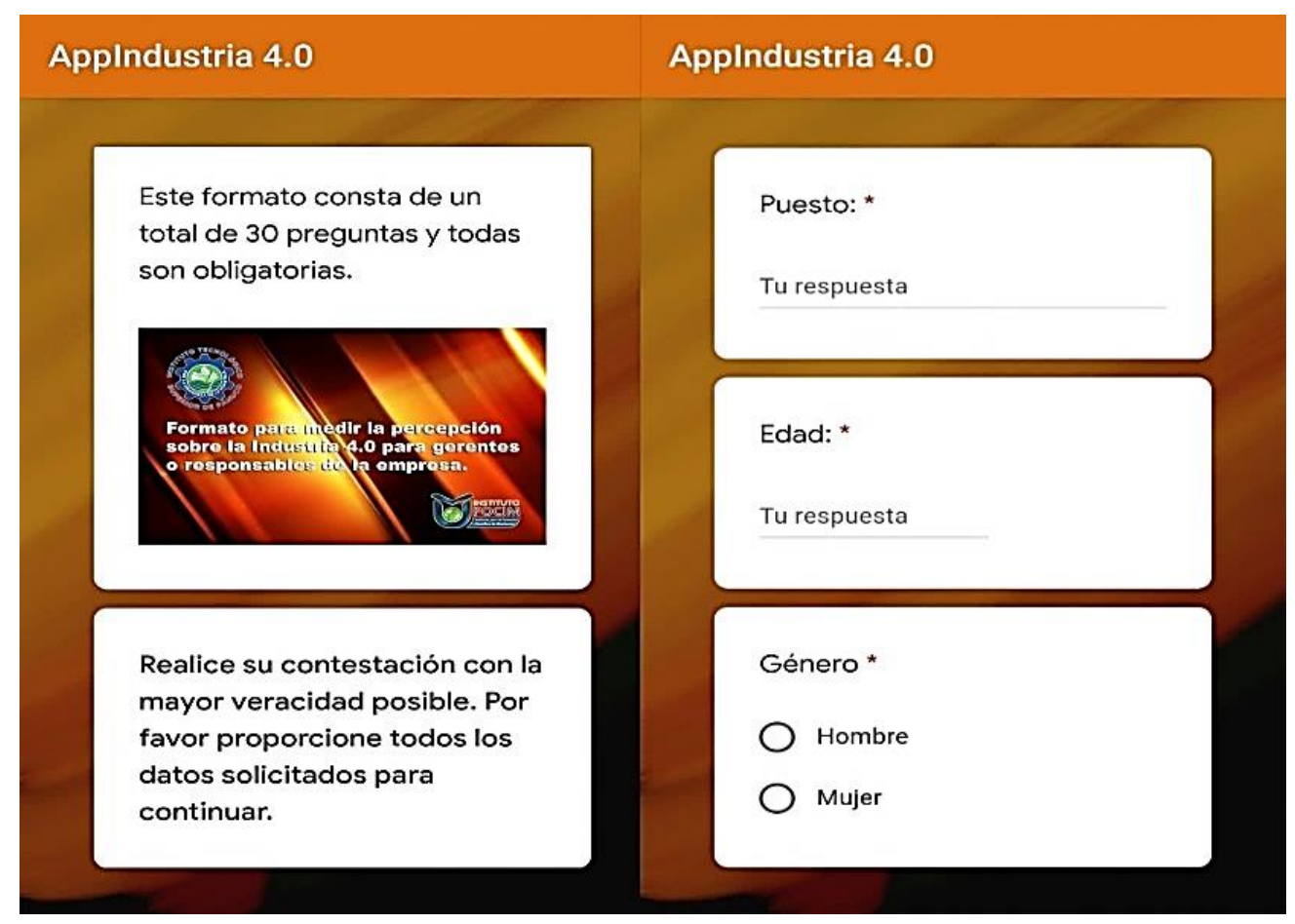

Fig. 1: Pantalla de bienvenida 
El primer componente de la aplicación es la variable de procesos integrada por cinco preguntas, en cuanto a las opciones para responder se utiliza la escala de Likert, en esta se integran valores que van del 1 al 7 . En el cuál, 1 es el nivel más bajo (Totalmente en desacuerdo) y 7 el nivel más alto (Totalmente de acuerdo). Para el procedimiento de contestación, no se puede pasar al siguiente componente si el primero no se contestó por completo. En este componente se intenta ubicar el nivel de conocimientos sobre los procesos de la empresa, si existe organización y supervisión de ellos y si son considerados como estrategia de mejora de la empresa. Las preguntas que integran este componente son: ¿En qué nivel ubica su conocimiento sobre procesos productivos que oferta su empresa?; ¿En qué nivel supervisa los procesos productivos de su empresa?; ¿En qué nivel de frecuencia ubica interrupciones en el proceso productivo de su empresa?; ¿En qué nivel de innovación se encuentra el proceso productivo que ofrece su empresa? y ¿Está usted de acuerdo que los procesos productivos que ofrece su empresa se pueden mejorar? (ver figura 2).
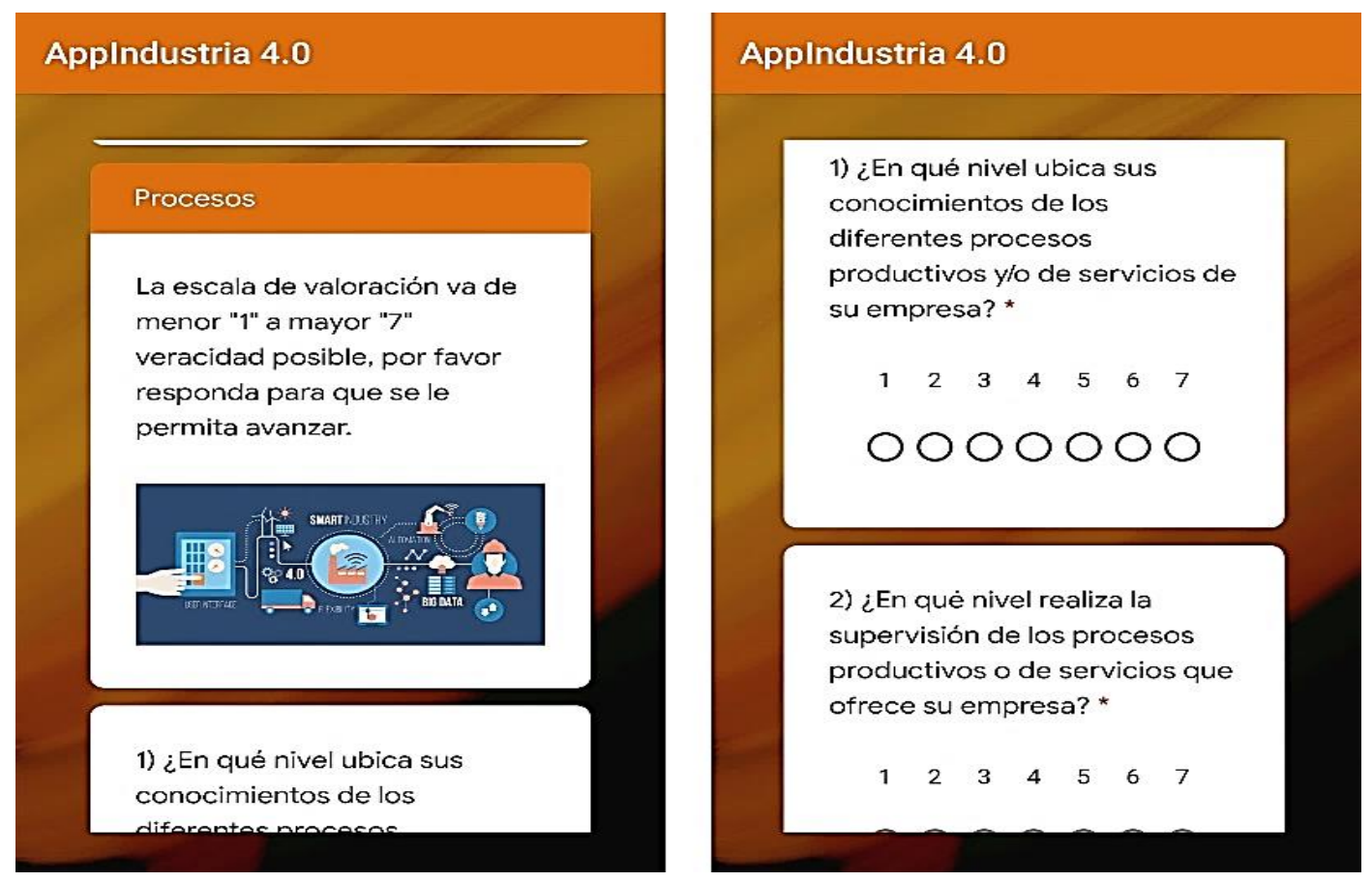

Fig. 2: Pantalla de evaluación de la variable de procesos

Para el segundo componente indicado como variable de producto, este se integra por cinco preguntas y utiliza una escala tipo Likert con el mismo orden del primer componente. En este componente se identifica como se percibe la mejora continua en la vida de un producto, si planea la mejora de sus productos y si las tecnologías utilizadas como la nube y sistemas de información traen beneficios en sus productos. Las preguntas que integran este componente son: ¿En qué nivel considera que la mejora continua incrementa el periodo de vida de un producto o servicio?; ¿Considera implementar tecnología en su empresa para mejorar estándares de competitividad de productos o servicios?; ¿identifica que su empresa tiene un producto o servicio estrella?; ¿Considera Usted que las tecnológicas de fabricación (robótica, software, realidad virtual, impresión 3D) han traído mejoras en los productos o servicios ofertados en su empresa? y ¿Considera que la nube (Google Drive, Dropbox) ha traído beneficios para diseñar, almacenar e incluso fabricar nuevos productos?

El tercer componente es la variable de personas, esta se integra por cinco preguntas y se utiliza la escala tipo Likert como opción de respuesta. En este componente se identifica la idoneidad de las personas en áreas de producción, si se tiene personal con la formación necesaria para adaptarse a los cambios tecnológicos. Las preguntas que integran este componente son: ¿Está Usted de acuerdo con la productividad del personal de su empresa es la adecuada?; ¿Considera Usted que el personal está comprometido con las estrategias de mejora implementadas en su empresa?; ¿Está Usted de acuerdo en capacitar al personal en uso y manejo de tecnologías para mejorar su modelo de negocio?; ¿Ubica Usted dentro de la plantilla de personal alguien con formación orientada en tecnología? y ¿En qué nivel ubica la capacitación del personal de su empresa para mejorar procesos productivos o servicios ofertados?

Para el cuarto componente de análisis la variable tecnología, en este componente se identifica el nivel de conocimiento e implementación de la tecnología de proceso, así como la perspectiva de su implementación para mejorar la competitividad e integración en la estrategia de negocios de la empresa. Las preguntas integradas en este componente son: ¿Está Usted de acuerdo en adquirir nueva tecnología para mejorar la producción y la competitividad de su empresa?; ¿Usted conoce los términos de tecnología básica, intermedia 
y estratégica?; ¿Identifica Usted si la institución tiene presupuesto asignado para la implementación de tecnología?; ¿Considera Usted que su empresa tiene el ancho de banda de Internet adecuado a sus necesidades? y ¿Considera Usted que su empresa utiliza los medios electrónicos de difusión como un diferenciador de competitividad? (ver figura 3).
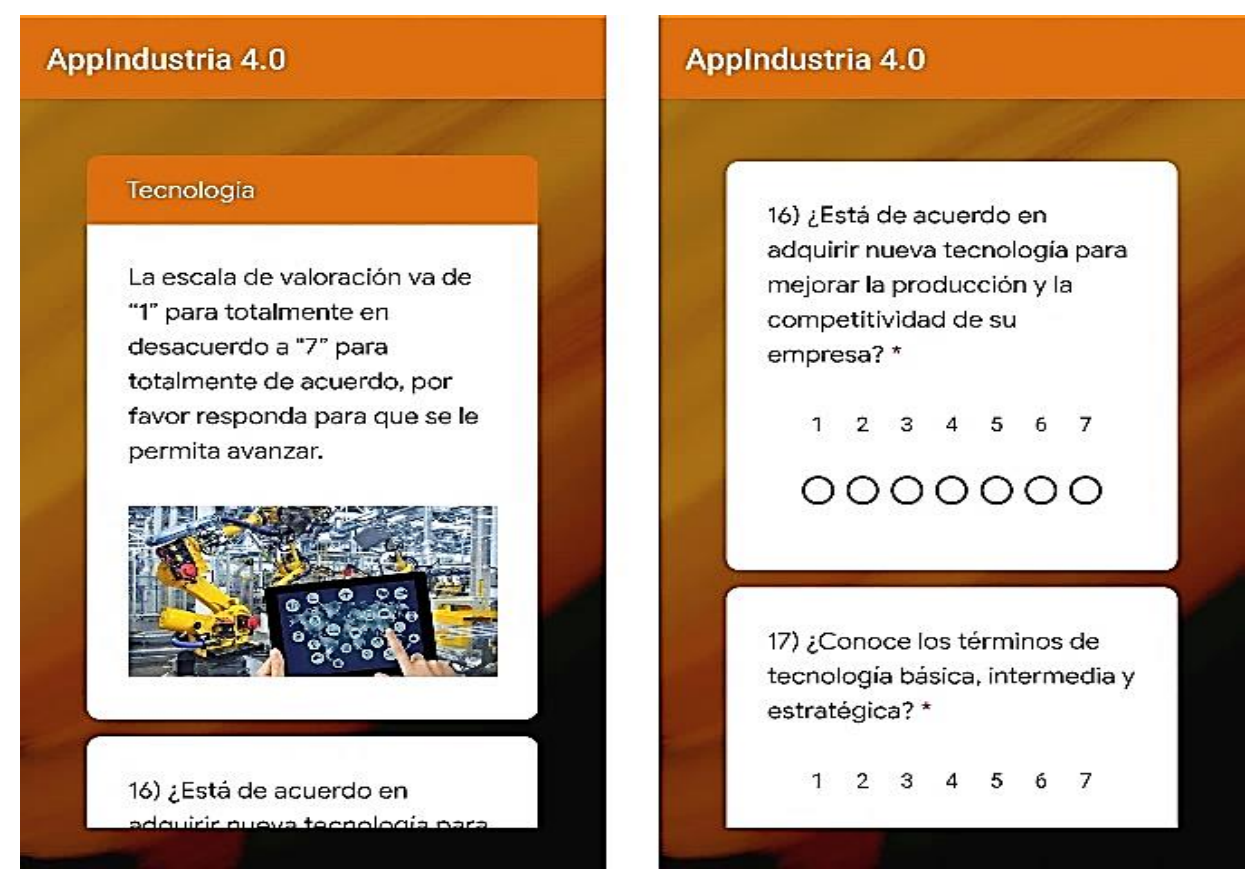

Fig. 3: Pantalla de evaluación de la variable de tecnología.

El quinto componente es la variable negocios, En esta variable se identifica la visión global de la empresa con respecto a la ventaja competitiva de utilizar tecnología 4.0 para su modelo de negocio. Las preguntas integradas en este componente son: ¿Indique el nivel de implementación de tecnología en su empresa que facilite las compras de sus clientes?; ¿Está usted de acuerdo en hacer una propuesta novedosa de base tecnológica como ventaja competitiva de su empresa?; ¿Considera usted que la industria 4.0, puede ser significativa para el desarrollo económico de la empresa?; ¿Indique en qué nivel está de acuerdo que la tecnología debe ser parte estratégica de su modelo de negocio?; y ¿Está usted de acuerdo que la empresa está obligada a transformarse estratégicamente para crear nuevos productos, servicios y modelos de negocio orientados a un nuevo tipo de cliente?

El sexto componente es la variable responsabilidad social, con este componente se completa el instrumento de medición que permite evaluar la percepción sobre el nivel de implementación de la industria 4.0 en las organizaciones. Esta variable se identifica el nivel de compromiso de la empresa con la responsabilidad social, la participación que tiene con el desarrollo sustentable y las acciones que emprende en su entorno. Las preguntas integradas en este componente son: ¿Está usted de acuerdo que los procesos utilizados en su empresa están armonizados para no afectar el medio ambiente?; ¿En qué nivel su personal realiza actividades vinculadas con la cultura ambiental dentro y fuera de la empresa?; ¿En su empresa se destinan recursos orientados a sensibilizar la importancia del medio ambiente en sus trabajadores?; ¿En qué nivel considera que su empresa respeta la normatividad en materia de seguridad industrial y medio ambiente?; y ¿Los residuos o desechos derivados de la producción de bienes o servicios están controlados y son resguardados? (ver figura 4).

\section{RESULTADOS}

Las aplicaciones móviles deben ser sometidas a evaluación estadística para garantizar la consistencia interna de los datos. Esta aplicación "App Industria 4.0" también completó esta metodología estadística para comprobar la fiabilidad y validez. Para este efecto, se calculó la varianza promedio extraída (AVE, por Average Variance Extracted). El $80 \%$ de los resultados se encuentran por encima del 0.50 recomendado como valor mínimo (Hair et al., 2017). 

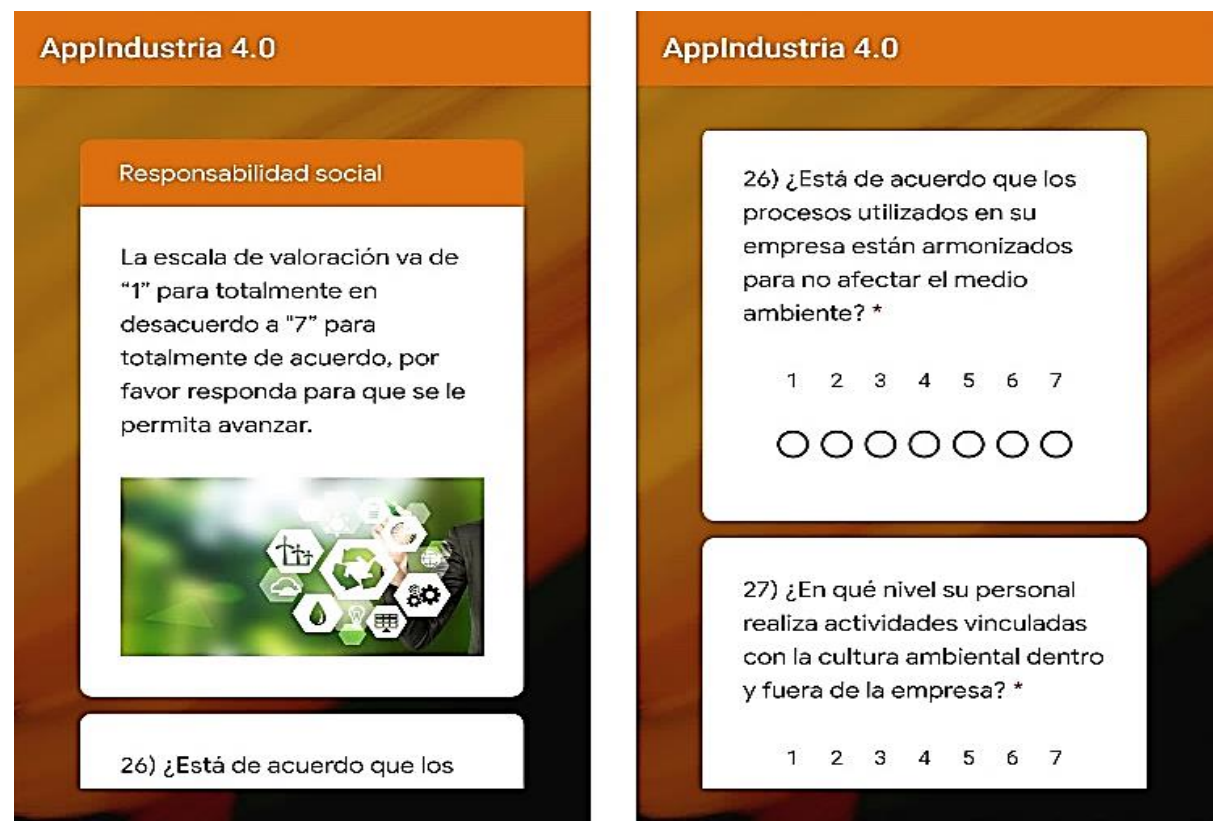

Fig. 4: Pantalla de evaluación de la variable de responsabilidad social.

De igual manera se realizó la estimación del factor de la inflación de la varianza (VIF, Variance Inflation Factor) que indica que no existe multicolinealidad entre pares de variables o factores independientes (ver Tabla 1); se obtuvo un valor máximo de 4.58 (Chen, 2011). El diseño del instrumento presentado es un acercamiento al sector empresarial para medir la percepción sobre el nivel de implementación de la industria 4.0. Para la validación del instrumento se aplicó una prueba piloto y se sometió a prueba mediante el análisis de alfa de Cronbach donde los resultados indican que es un instrumento estable ya que el $80 \%$ de los resultados están por encima de 0.70 (Domínguez, 2012).

Tabla 1: Resultados de fiabilidad, validez e inflación de las variables

\begin{tabular}{|c|c|c|c|c|c|c|c|}
\hline \multicolumn{2}{|c|}{ Constructos de investigación } & Media & Desviación estándar & $\begin{array}{c}\text { Alfa } \\
\text { Cronbach }\end{array}$ & AVE & VIF & $\begin{array}{l}\mathrm{KMO} \\
\text { Bartlett }\end{array}$ \\
\hline \multirow{4}{*}{ Procesos } & Proc1 & 5.77 & 1.341 & \multirow{4}{*}{0.720} & \multirow{4}{*}{0.553} & \multirow{4}{*}{1.584} & \multirow{4}{*}{.742} \\
\hline & Proc2 & 5.76 & 1.287 & & & & \\
\hline & Proc3 & 6.18 & 1.073 & & & & \\
\hline & Proc4 & 6.23 & 1.015 & & & & \\
\hline \multirow{4}{*}{ Persona } & Pers11 & 5.98 & 1.374 & \multirow{4}{*}{0.761} & \multirow{4}{*}{0.608} & \multirow{4}{*}{4.304} & \multirow{4}{*}{.647} \\
\hline & Pers12 & 6.30 & 1.030 & & & & \\
\hline & Pers14 & 5.47 & 1.844 & & & & \\
\hline & Pers15 & 5.82 & 1.849 & & & & \\
\hline \multirow{4}{*}{ Tecnología } & Tecn16 & 5.98 & 1.401 & \multirow{4}{*}{0.665} & \multirow{4}{*}{0.488} & \multirow{4}{*}{1.871} & \multirow{4}{*}{.626} \\
\hline & Tecn17 & 5.96 & 1.355 & & & & \\
\hline & Tecn18 & 6.02 & 1.235 & & & & \\
\hline & Tecn20 & 6.03 & 1.327 & & & & \\
\hline \multirow{5}{*}{ Negocios } & Nego21 & 5.71 & 1.629 & \multirow{5}{*}{0.801} & \multirow{5}{*}{0.567} & \multirow{5}{*}{4.588} & \multirow{5}{*}{.790} \\
\hline & Nego22 & 5.90 & 1.546 & & & & \\
\hline & Nego23 & 6.06 & 1.326 & & & & \\
\hline & Nego24 & 6.28 & 1.131 & & & & \\
\hline & Nego25 & 6.37 & 1.166 & & & & \\
\hline \multirow{4}{*}{$\begin{array}{l}\text { Responsabili } \\
\text { dad Social }\end{array}$} & Resp27 & 6.21 & 1.329 & \multirow{4}{*}{0.848} & \multirow{4}{*}{0.687} & \multirow{4}{*}{$\begin{array}{c}\text { Variable } \\
\text { dependiente }\end{array}$} & \multirow{4}{*}{.792} \\
\hline & Resp28 & 6.22 & 1.405 & & & & \\
\hline & Resp29 & 6.35 & 1.333 & & & & \\
\hline & Resp30 & 6.31 & 1.338 & & & & \\
\hline
\end{tabular}


Para fortalecer el análisis de los datos recuperados con la App Industria 4.0, se utilizó un software estadístico XLSTAT, se integraron las variables: Procesos, Producto, Personas, Tecnología, Negocios y Responsabilidad social y se correlacionaron entre sí. Para este ejemplo se identificó el impacto y contribución de la variable Responsabilidad Social (RespSocial) como variable dependiente o variable de salida y todas las demás como variables de entrada o independientes. De forma adicional, se pretende presentar una metodología de análisis de consistencia de datos internos de un instrumento de medición, para futuros trabajos que estén utilizando aplicaciones como instrumento de medición. Los resultados que se presentan ejemplifican el potencial de análisis con los datos encontrados.

\section{Resultados del modelo de ruta y cargas de factores}

Los resultados de la estimación de PLS para el modelo estructural, así como las cargas de las variables de investigación como son: Procesos, Producto, Personas, Tecnología, Negocios y Responsabilidad Social. Derivado de las pruebas de validez se ajustó (no se consideró) una variable para este ejercicio "Producto" (p6-p10), También se ajustó el número de ítems de algunas variables (p5, p13, p19 y p26) y sólo se consideraron aquellos valores superiores a 0.50 , lo que significa que estos valores explicaron más del $50 \%$ de la varianza y alcanzaron el umbral igual o superior a 0.50. Con esta acción, se permitió replantear las preguntas y volver analizar los datos de contribución entre variables (ver figura 5).

Las variables de la aplicación analizada indican una fuerte relación entre la variable tecnología y la responsabilidad social con una aportación del $44.09 \%$; los procesos y la responsabilidad social con una aportación del $14.01 \%$, tan sólo entre estas dos variables explican el $58.1 \%$ de la relación existente. Las variables que más aportaron al proceso fueron las variables de tecnología y personas. En este ejemplo se identifican los resultados encontrados mediante ecuaciones estructurales PLS con software XLSTAT, los datos mostrados son de correlación, coeficiente de trayectoria y la contribución que hace cada variable para explicar su relación con la variable RespSocial. Con los datos que realiza el XLSTAT, se pueden probar hipótesis, ubicar la matriz de correlación, correlación cruzada, pesos de los ítems, así como la estadística descriptiva. La estadística indicada anteriormente fortalece el método planteado para analizar los componentes de la aplicación Applndustria 4.0.

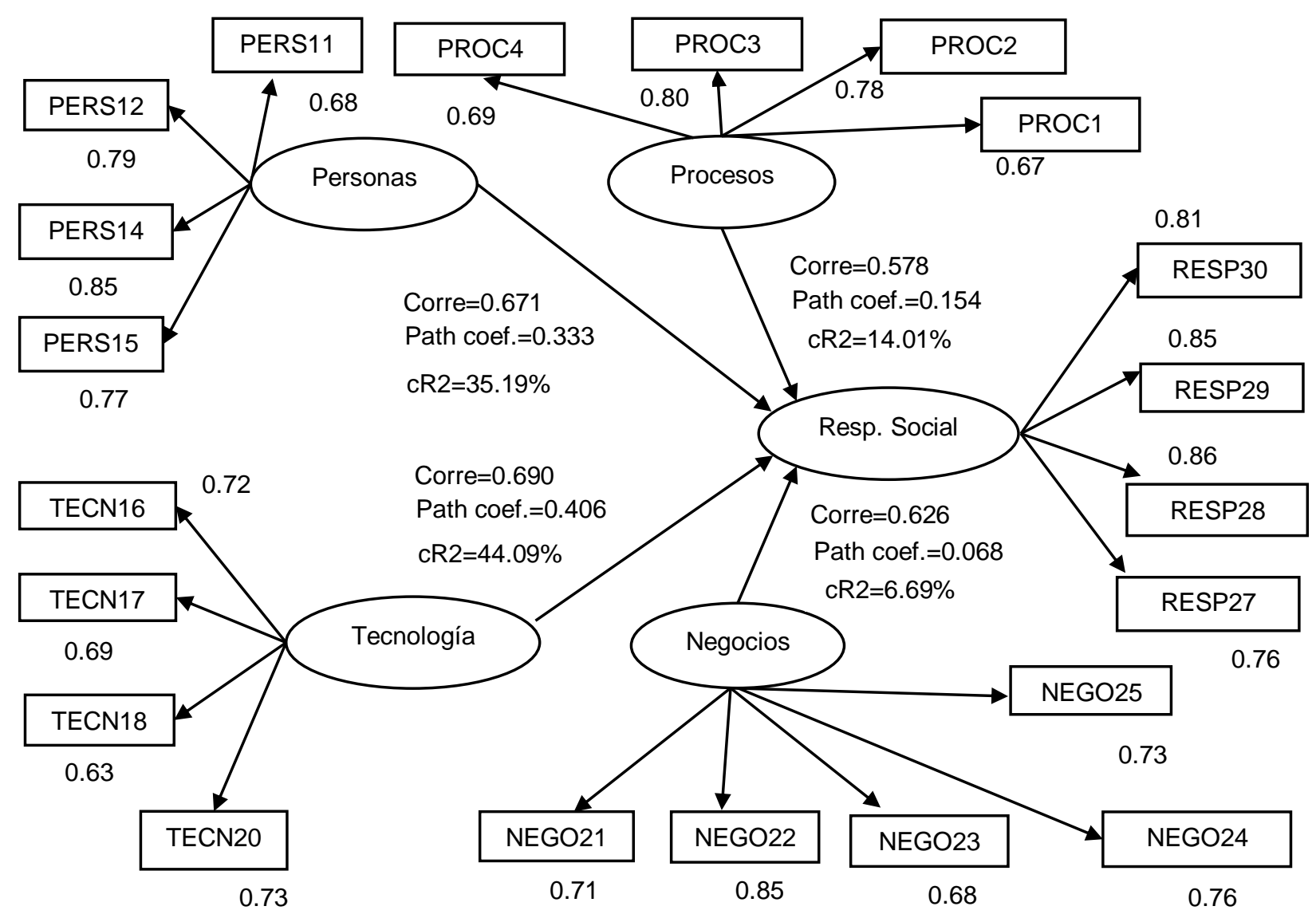

Fig. 5: Contribución de cargas de las variables. 
Tabla 2. Resultados del análisis del modelo de ecuación estructural

\begin{tabular}{|c|c|c|c|l|}
\hline \multicolumn{1}{|c|}{ Path } & Hipótesis & $\begin{array}{c}\text { Path Coeficiente } \\
(\beta)\end{array}$ & t-estadística & \multicolumn{1}{c|}{ Decisión } \\
\hline Procesos $\rightarrow$ Resp. social & $\mathrm{H}_{1}$ & 0.154 & 1.744 & Se acepta $\mathrm{H}_{0}$ \\
\hline Personas $\rightarrow$ Resp. social & $\mathrm{H}_{2}$ & 0.333 & 2.818 & Se rechaza $\mathrm{H}_{0}$ \\
\hline Tecnología $\rightarrow$ Resp. social & $\mathrm{H}_{3}$ & 0.406 & 4.528 & Se rechaza $\mathrm{H}_{0}$ \\
\hline Negocios $\rightarrow$ Resp. social & $\mathrm{H}_{4}$ & 0.068 & 0.576 & Se acepta $\mathrm{H}_{0}$ \\
\hline
\end{tabular}

Las pruebas de hipótesis están determinadas por los coeficientes de trayectoria (Path coeficiente), así como por los valores de t obtenidos en el modelo estructural. Las pruebas t de dos colas, se llevaron con un nivel de significancia del $95 \%$. De acuerdo con Martínez (2018), los coeficientes de trayectoria de las variables del modelo y los valore t, determinan la relación de significancia entre las variables del modelo. Los resultados de las distintas pruebas de hipótesis se muestran en la Tabla 3. En la parte que corresponde a la aplicación móvil Appindustria4.0.apk, soportado por el sistema operativo (S.O) Android que incorpora la lógica del servicio dirigida a personal directivo de las organizaciones. En este estudio la mayoría de las aplicaciones están enfocadas al conocimiento de la industria 4.0, se presenta una aplicación móvil que evalúa el comportamiento de las organizaciones con respecto al nivel de aplicación de la industria 4.0., se tiene un enfoque de carácter tecnológico y organizacional, ya que el uso de esta herramienta tecnológica será capaz de ayudar a las organizaciones para evaluarse en cualquier parte del mundo.

Tabla 3: Resultados de la prueba de hipótesis

\begin{tabular}{|c|l|}
\hline $\begin{array}{c}\text { Prueba de } \\
\text { hipótesis }\end{array}$ & \multicolumn{1}{c|}{ Resultado } \\
\hline 1 & $\begin{array}{l}\text { El Proceso tiene una influencia positiva en la Responsabilidad Social, en este estudio se propuso esta } \\
\text { hipótesis. En la Tabla 2 se pueden observar los valores de }(\beta=0.154) \text { y de }(\mathrm{t}=1.744) \text { que explican la poca } \\
\text { significancia entre estas dos variables. Este resultado sugiere que los Procesos en la empresa no influyen } \\
\text { de forma significativa en la Responsabilidad Social, se acepta } \mathrm{H}_{0} .\end{array}$ \\
\hline 2 & $\begin{array}{l}\text { Las Personas tienen una influencia positiva en la Responsabilidad Social, en este estudio se propuso } \\
\text { esta hipótesis. En la Tabla 2 se pueden observar los valores de }(\beta=0.333) \text { y de (t=2.818) que explican } \\
\text { que hay significancia entre estas dos variables. Este resultado sugiere que las personas en la empresa } \\
\text { influyen de forma positiva en la Responsabilidad Social, se rechaza } \mathrm{H}_{0} .\end{array}$ \\
\hline 3 & $\begin{array}{l}\text { La Tecnología tiene una influencia positiva en la Responsabilidad Social, en este estudio se propuso esta } \\
\text { hipótesis. En la Tabla } 2 \text { se pueden observar los valores de }(\beta=0.406) \text { y de (t=4.528) que explican que } \\
\text { hay significancia entre estas dos variables. Este resultado sugiere que la Tecnología de la empresa } \\
\text { influye de forma positiva en la Responsabilidad social, se rechaza } \mathrm{H}_{0 .}\end{array}$ \\
\hline 4 & $\begin{array}{l}\text { La variable Negocios tiene una influencia positiva en la Responsabilidad Social de la empresa, en este } \\
\text { estudio se propuso esta hipótesis. En la Tabla } 2 \text { se pueden observar los valores de }(\beta=0.068) \text { y de } \\
(\mathrm{t}=0.576) \text { explican la poca significancia entre estas dos variables. Este resultado sugiere que Negocios } \\
\text { no influyen de forma significativa en la Responsabilidad Social, se acepta } \mathrm{H}_{0 .} .\end{array}$ \\
\hline
\end{tabular}

\section{DISCUSIÓN}

La industria 4.0 ofrece una gran oportunidad para alinear objetivos del desarrollo sostenible con la transformación digital en las organizaciones (Beier et al., 2020; Bai et al., 2020). Se considera relevante tener nueva tecnología, identificar barreras existentes, comprender desafíos de la industria 4.0; así como, obtener beneficios sociales, económicos y ambientales (Abdul et al., 2020). Para Kumar et al. (2020), la sustentabilidad de las operaciones en las pequeñas y medianas empresas se encuentran bajo presión; el tener una aplicación que puedan evaluar el nivel de implementación de la industria 4.0, es un área de oportunidad para las empresas. Las aplicaciones móviles se están utilizando en aspectos del aprendizaje y capacitación, esto hace sentido con la propuesta de una aplicación empresarial AppsIndustria 4.0 para evaluar el nivel de implementación de la industria 4.0.

Las aplicaciones móviles permiten que las personas desarrollen conocimiento de diferentes maneras y se construya el entendimiento entre las personas; la tecnología móvil con frecuencia cambia el patrón de actividad de aprendizaje y trabajo; el aprendizaje móvil indica que debe ocurrir una interacción entre el aprendizaje y la presencia social; Kuhn esta parte Grané (2016) indica que para desarrollar un modelo fiable y eficaz para seleccionar aplicaciones que aseguren contenidos para diferentes edades de usuarios es un objetivo necesario y ambicioso. En este sentido, la tecnología de acuerdo con Lin y Doughty (2015) indican que se deben utilizar la estadística para analizar la consistencia interna de los datos en los instrumentos de medición de aplicaciones móviles. El instrumento de medición presentado aprobó las pruebas de validez para un instrumento de medición, así como la Applndustria 4.0. 
Las tecnologías móviles han traído beneficios en diferentes áreas del conocimiento, el entendimiento de necesidades del mercado para responder inquietudes como el de parejas que presentan en el proceso de fertilidad (Ali et al., 2020). También existen modelos que han sido utilizados en la industria del plástico, donde tener un modelo de evaluación de la industria 4.0 es altamente potencial para lograr un desarrollo sostenible; es una realidad que las aplicaciones deben tener diferentes etapas de evaluación para acoplar aspectos y cumplir objetivos para lo cual fueron creadas (Benitez et al., 2020). De esta forma, los usos de las Apps se están generalizando y diversificando, las áreas de análisis han manifestado resultados positivos, pero requieren análisis y validación de sus datos. Los resultados obtenidos en este trabajo identifican esa parte que se tiene que hacer con los instrumentos de evaluación por aplicaciones móviles.

En los resultados en el modelo de trayectorias y cargas factoriales se puede indicar que las variables con mayor contribución que explican la relación entre ellas son Procesos-RespSocial con 24.87 \%; PersonasRespSocial con $24.11 \%$ y la Tecnología-RespSocial con $42.33 \%$. En cuanto a las cargas factoriales sólo se incluyeron en las itéms que se ubicaron por arriba del mínimo recomendado de 0.5 (Rosipal y Kromer, 2006). Con respecto a la Applndustria4.0, se integró con todas las variables e itéms completos, derivado de las pruebas estadísticas realizadas y los ajustes realizados para su liberación.

\section{CONCLUSIONES}

Los resultados muestran que la Apps Industria 4.0 es factible de utilizar para las organizaciones que desean medir el nivel de implementación de la organización. La aplicación móvil acreditó las pruebas de consistencia interna de los datos. Las aplicaciones móviles se han generalizado en los últimos tiempos, esto permite hacer una reflexión sobre la evaluación interna de cada una de ellas, esta acción permitirá mantener su vigencia y uso; la aplicación móvil propuesta no escapa a esta revisión.

Se identificó una relación positiva entre las variables del ejemplo propuesto, esto indica que hay una correlación fuerte entre ellas, las variables que indicaron mayor contribución en esta relación son: procesos, personas y tecnologías. Las variables con menor participación negocios y producto presentaron menor aportación. El instrumento fue sometido a revisión para identificar debilidades en algunos ítems que fueron ajustados en la aplicación móvil propuesta para dejarla apunto. Una aplicación de este tipo es una innovación tecnológica ya que no existe hasta el momento una que mida la industria 4.0, que esté desarrollada en Android y que se encuentre hospedada en la Play Store para su descarga.

Finalmente, la Industria 4.0 podría generar cambios en la fuerza laboral de las organizaciones; medir la percepción de cómo los trabajadores miden su nivel de implementación es una buena estrategia para la toma de decisiones. La evaluación de las aplicaciones en cuanto a su confiabilidad estadística es un buen punto para que sea considerada por sobre las existentes. Su creciente utilización permitirá decidir al usuario en qué tipo de aplicación móvil podría obtener beneficio y cuál no. Las condiciones cambiantes del entorno empresarial se diversifican, los modelos de negocio y las aplicaciones móviles que quieran permanecer vigentes se tendrán que adaptar a nuevos retos.

\section{REFERENCIAS}

Abdul, A.Q., Helmi A.M., y otros 3 autores, Impeding challenges on industry 4.0 in circular economy- palm oil industry in Malaysia, https://doi.org/10.1016/j.cor.2020.105052, Computers \& Operations Research, 123, 1-14 (2020)

Ali, R., Gürtin, Z.B., y Harper, J.C., Do fertility tracking applications offer women useful information about their fertile window?, https://doi.org/10.1016/j.rbmo.2020.09.005, Journal Reproductive Biomedicine Online, 42 (1), 273-281 (2020)

Bai, C., Dallasega, P., Orzes, G., y Sarkis, J., Industry 4.0 technologies assessment: a sustainability perspective, https://doi.org/10.1016/j.ijpe.2020.107776, International Journal of Production Economics, 229, 1-15 (2020)

Beck, K., Beedle, M., y otros 15 autores, Manifesto for agile software development, Utah: The Agile Alliance (2001)

Beier, G., Ullrich, A., y otros 3 autores, Industry 4.0-How it is defined from a sociotechnical perspective and how much sustainability it includes, https://doi.org/10.1016/j.jclepro.2020.120856, Journal of Cleaner Production, 259, 1-13, (2020)

Benitez, E.O., Becker, C.M., y otros 5 autores, Expected impact of industry 4.0 technologies on sustainable developmentA study in the context of Brazil's plastic industry, https://doi.org/10.1016/j.spc.2020.07.018, Sustainable Production and Consumption, 25, 102-122 (2020)

Bueno, A., Godinho, F.M., y Frank, A.G., Smart production planning and control in the industry 4.0 context: a systematic literature review, https://doi.org/10.1016/j.cie.2020.106774, Computers \& Industrial Engineering, 149, 1-21 (2020)

Chavira, G.J., y Arredondo, A.A., Aplicaciones móviles como herramientas en los servicios de salud, https://doi.org/10.19136/hs.v16n2.1498, Horizonte Sanitario, 16 (2), 85-91 (2017) 
Chen, S., y Fan, J., Measuring corporate social responsibility based on a fuzzy analytical hierarchy process, https://doi.org/10.5815/ijcnis.2011.05.02, International Journal Computer Network and Information Security, 5, 13-22 (2011)

Corallo, A., Lazoi, M., y Lezzi, M., Cybersecurity in the context of industry 4.0: a structured classification of critical assets and business impacts, https://doi.org/10.1016/j.compind.2019.103165, Computers in Industry, 114, 1-15 (2020)

De Giovanni, P., y Cariola, A., Process innovation through industry 4.0 technologies, lean practices and green supply chains, https://doi.org/10.1016/j.retrec.2020.100869, Research in Transportation Economics, ISSN: 0739-8859 (2020)

Domínguez, S., Propuesta para el cálculo de alfa ordinal y theta de Armor, https://doi.org/10.15381/rinvp.v15i1.3684, Revista de Investigación en Psicología, 15(1), 213-217 (2012)

Faraz, M., y Petraite, M., Industry 4.0 technologies, digital trust and technological orientation: what matters in open innovation?, https://doi.org/10.1016/j.techfore.2020.120332, Technological Forecasting and Social Change, 161, 1-11 (2020)

Fernández, A., Cunha, J., y otros 3 autores, Research and development project assessment and social impact, http://dx.doi.org/10.1590/0103-6513.110212, Journal Production, Vol. 25 (4), 725-728 (2015)

Garita, R.A., Tecnología móvil-desarrollo de sistemas y aplicaciones para las unidades de información, https://doi.org/10.15517/eci.v3i2.10654, E-Ciencias de la Información, 3 (2), 1-14 (2013)

Gaudencio, P., Coelho, A., y Ribeiro, N., The role of trust in corporate social responsibility and worker relationships, https://doi.org/10.1108/JMD-02-2016-0026, Journal of Management Development, Vol. 36 (4), $478-492$ (2017)

Grané, O.M., y Crescenzi, L.L., Modelo teórico para el diseño y evaluación de la calidad en las apps infantiles (0-8 años), https://doi.org/10.1344/der.2016.29.227-245, Digital Education Review, 29, 227-245 (2016)

Hair, J.F., Hult, T.M., Ringle, C., y Sarstedt, M., A primer on partial least squares structural equation modeling (PLS-SEM), $2^{\mathrm{a}}$ edition, SAGE publishing, Los Angeles (2017)

Hsion, L.C., Lim, S., y otros 5 autores, A review of industry 4.0 revolution potential in a sustainable and renewable palm oil industry-HAZOP, https://doi.org/10.1016/j.rser.2020.110223, Renewable and Sustainable Energy Reviews, 135, 1-19 (2020)

Kadir, B., y Broberg, O., Human-centered design of work systems in the transition to industry 4.0, https://doi.org/10.1016/j.apergo.2020.103334, Applied Ergonomics, 92, 1-14 (2021)

Kumar, R., Singh, R.K., y Dwivedi, Y.K., Application of industry 4.0 technologies in SMEs for ethical and sustainable operations-analysis of challenges, https://doi.org/10.1016/j.jclepro.2020.124063, Journal of Cleaner Production, 275, 1-13 (2020)

Lin, P., y Doughty, T., Developing an app evaluation rubric for practitioners in special education. Journal of Special Education Technology, 30 (1), (2015)

Mantilla, M., Camargo, L., y Medina, B., Metodología para el desarrollo de aplicaciones móviles. Tecnura-tecnología y cultura afirmando el conocimiento, 18 (40), 20-35 (2014)

Martínez, M., Aplicación de la técnica PLS-SEM en la gestión del conocimiento: un enfoque técnico práctico, https://doi.org/10.23913/ride.v8i16.336, Revista Iberoamericana para la Investigación y el Desarrollo Educativo, 8 (16), 135, (2018)

Rosipal, R., y Krämer, N., Overview and recent advances in partial least squares, https://doi.org/10.1007/11752790_2, Springer-Lecture Notes in Computer Science, 3940, 34-51 (2006)

Ruiz, H.S., Díaz, M.A., y Ruiz, H.S., The perception of industry 4.0 in the managers of automobile distribution companies https://doi.org/10.24052/JBRMR/V14IS01/ART-07, Journal of Business and Retail Management Research (JBRMR), 14 (1), 71-78 (2019)

Sánchez, M., Exposito, E., y Aguilar, J., Industry 4.0: survey from a system integration perpective, https://doi.org/10.1080/0951192X.2020.1775295, International Journal of Computer Integrated Manufacturing, 33 (10), 125 (2020)

Shayganmehr, M., Kumar, A., Garza, J., y Moktadir, A., Industry 4.0 enablers for a cleaner production and circular economy within the context of business ethics: a study in a developing country, https://doi.org/10.1016/j.jclepro.2020.125280, Journal of Cleaner Production, 281, 1-46 (2021)

Silvestri, L., Forcina, A., y otros 3 autores, Maintenance transformation through industry 4.0 technologies: asystematic literature review, https://doi.org/10.1016/j.compind.2020.103335, Computers in Industry, 123, 1-16 (2020)

Sony, M., y Naik, S., Critical factors for the successful implementation of industry 4.0: a review and future research direction, https://doi.org/10.1080/09537287.2019.1691278, Production Planning \& Control, 31 (10), 799-815 (2020) 